\title{
Research Paper \\ Investigating the Causes of Death in the Injured of Traffic Accidents Referred to Vali-Asr Hospital in Arak, Based on Oral Autopsy and Forensic Autopsy
}

\author{
*Mohammad Jamalian ${ }^{1} \odot$, Mohsen Eslamdost ${ }^{1}$, Ali Rezaee ${ }^{1}$, Shabanali Alizadeh ${ }^{2} \odot$
}

1. Department of Forensic Medicine, School of Medicine, Arak University of Medical sciences, Arak, Iran.

2. Department of Surgery, School of Medicine, Arak University of Medical sciences, Arak, Iran.

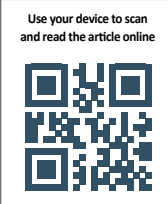

ctition: Jamalian M, Eslamdost M, Rezaee A, Alizadeh S. [Investigating the Causes of Death in the Injured of Traffic Accidents Referred to Vali-Asr Hospital in Arak, Based on Oral Autopsy and Forensic Autopsy (Persian)]. Journal of Arak University of Medical Sciences (JAMS). 2020; 23(3):338-347. https://doi.org/10.32598/JAMS.23.3.5986.1

https://doi.org/10.32598/JAMS.23.3.5986.1

Key words: Road traffic injuries, Death, Accident

\section{A B STRACT}

Article Info:

Received: 18 Sep 2019

Accepted: 26 May 2020

Available Online: 01 Aug 2020

Background and Aim The deaths caused by traffic accidents are one of the three leading causes of death in Iran. Considering that all deaths from traffic accidents in the forensic organization are undergoing autopsy and also in the treatment centers under oral autopsies, the cause of death is determined, we determined that the cause of reported death from oral autopsy and medical autopsy Compare the law. Methods \& Materials In this descriptive study, patients who were admitted to the forensic medical center during the year 1395 admitted to the Vali-e-Asr Hospital in Arak, were referred. The causes of death in Vali-asr Hospital were evaluated and compared with the outcome of the forensic oral hearing.

Ethical Considerations This study ethcally approved by the ethics committee of the Arak University of Medical Sciences (Code: IR.ARAKMU.REC.1395.226).

Results In this study, 109 patients (1.33\%) died from a total of 8153 patients with traffic accidents referred to Valiasr hospital in Arak. Of these, $68.8 \%$ were male and $31.2 \%$ were women. The Mean \pm SD age of the patients died was $44.03 \pm 22.43$ years. Also, $59.6 \%$ of the dead died during the first 24 hours of the accident. The most common cause of death, according to the results of oral autopsy, was "multiple injuries," accounting for $49.5 \%$ of the patients in 45 patients.

Conclusion Abundance of deaths among patients with road traffic accidents and they referred to Valiasr hospital, which was $1.33 \%$. According to the existing standards and considering that the mortality rate in this center is less than $1.5 \%$. It seems that the level of service delivery in this center is favorable.

\section{Extended Abstract}

\section{Introduction}

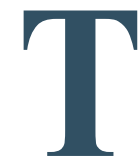

raffic accidents deaths occur either at the time of the accident or within 30 days due to injury caused by the accident [1]. By 2020, traffic accident injuries will be the second cause of disability in developing countries and the third cause of death worldwide [2].

Injuries due to traffic accidents are one of the three main causes of death in Iran. All cases of this type of death are autopsied in the Forensic Medicine Organization and the medical centers perform verbal autopsy and determine the cause of death. Therefore, in this study, we decided to inves-

\section{* Corresponding Author:}

Mohammad Jamalian, PhD.

Address: Department of Forensic Medicine, School of Medicine, Aral University of Medical sciences, Arak, Iran

Tel: +98 (86) 32222003

E-mail: m.jamaliyan@arakmu.ac.ir 
tigate the cause of death reported by verbal and forensic autopsy and compare the results.

\section{Materials and Methods}

This cross-sectional study included all patients who were admitted to Vali-e-Asr Hospital in Arak City during 2016 due to injuries caused by traffic accidents and were referred to a forensic medical center for autopsy after death. Inclusion criteria were the death of the hospitalized patient within 30 days after the traffic accident, and exclusion criteria were the death of the hospitalized patient after 30 days of the accident and no medical (clinical) autopsy. After the death of the traffic accident patient, verbal autopsy information (i.e., information obtained from the patient's history and case evidence) was collected and recorded. Due to the fact that all the bodies of traffic accidents are referred to forensic medicine to report the cause of death, medical autopsy information was also collected and recorded. Finally, a comparison was made between the results of these two types of autopsies.

\section{Results}

This cross-sectional study was performed in 2016. Out of 8153 traffic accident patients referred to Vali-e-Asr Hospital in Arak City, 109 cases resulted in death (1.33\%). Eighteen patients who did not undergo medical autopsy due to various causes were excluded from the study. The causes of death due to traffic accidents based on verbal and medical autopsy are shown in Tables 1 and 2.

The most common cause of death, according to the verbal autopsy results, was "multiple injuries", which occurred in $45(49.5 \%)$ patients. The second most common cause of death was "head trauma" which occurred in 28 (30.8\%) patients (Table 1).

The causes of death of patients in traffic accidents were examined after a forensic autopsy and it was found that the most common cause of death of patients in traffic accidents was head trauma, which was present in 42 patients (46.1\%) of the total patients. The second most common cause of death in these patients based on forensic autopsy was multiple fractures, which occurred in 33 patients $(36.2 \%)$ of the total patients (Table 2).

Table 1. Evaluation of the cause of death based on verbal autopsy

\begin{tabular}{|c|c|}
\hline Verbal Autopsy Result & No. (\%) \\
\hline Multiple injuries & $45(49.5)$ \\
\hline Head trauma & $28(30.8)$ \\
\hline Hypovolemic shock & $5(5.5)$ \\
\hline Pulmonary embolism & $5(5.5)$ \\
\hline Spinal cord injury & $4(4.4)$ \\
\hline Multi-organ failure & $1(1.1)$ \\
\hline Adult respiratory distress syndrome & $1(1.1)$ \\
\hline Tracheal stenosis & $1(1.1)$ \\
\hline Extensive myocardial infarction & $1(1.1)$ \\
\hline Total & $91(100)$ \\
\hline
\end{tabular}

Table 2. Cause of death based on patients' forensic autopsy

\begin{tabular}{cc}
\hline Forensic Autopsy Result & No. (\%) \\
\hline Head trauma & $42(46.1)$ \\
Multiple fractures & $33(36.2)$ \\
Bleeding & $8(8.8)$ \\
Other causes & $8(8.8)$ \\
Total & $91(100)$ \\
\hline
\end{tabular}


Comparison of the causes of death of patients in traffic accidents based on oral and forensic autopsies showed that the cause of death of these patients was the same in 51 cases $(56 \%)$ and was different in 40 patients $(44 \%)$. Forensic clinical autopsy data were not available in 18 patients and therefore their results could not be compared.

\section{Discussion}

Out of 8153 patients in traffic accidents referred to Valie-Asr Hospital in Arak City in 2016, a total of 109 patients (1.33\%) died. The most common cause of death, according to the verbal autopsy results, was "multiple injuries" in 45 patients (49.5\%). According to forensic autopsies, the most common cause of death of patients due to traffic accidents was head trauma, which was present in 42 patients (46.1\%) of the total number of patients. According to verbal and forensic autopsies, the causes of death of patients in traffic accidents were similar in 51 cases $(56 \%)$ and different in 40 cases (44\%).

The results of studies conducted by A Montazeri and NB Kumar showed that head trauma is the leading cause of death due to traffic accidents $[16,17]$. These results are consistent with the results of medical autopsy in the present study.

\section{Conclusion}

In general, the results of this study showed that the frequency of deaths among road accident victims who referred to Vali-e-Asr Medical Center in Arak City was $1.33 \%$. According to the existing standards and considering that the mortality rate in this center is less than $1.5 \%$, it seems that the quality of services in this center is desirable. The results of this study showed that most road accident patients lose their lives during the first 24 hours of the accident, which shows that most of these deaths are inevitable due to the severity of injuries.

\section{Ethical Considerations}

\section{Compliance with ethical guidelines}

This study ethcally approved by the ethics committee of the Arak University of Medical Sciences (Code: IR.ARAKMU.REC.1395.226).

\section{Funding}

This study financially supported by the Vice Chancellor for Research and Technology of Arak University of Medical Sciences.

\section{Authors' contributions}

Conceptualization, comparison of results: Mohammad Jamalian; Research design and collaboration in sampling: Mohsen Islamdoost, Ali Rezaei; Sampling: Shaban Ali Alizadeh; Text Review: All Authors

\section{Conflicts of interest}

The authors declared no conflict of interest.

\section{Acknowledgements}

The authors would like to thank the Vice Chancellor for Research and Technology of Arak University of Medical Sciences and the Vali-e-Asr Hospital Clinical Research Development Center. 


\title{
بررسى علل فوت در مجروحين حوادث ترافيكى مراجعه كننده به بيمارستان ولى عصر اراك بر اساس اتويسى شفاهى و اتويسى يزئشكى قانونى
}

\author{
"سيد محمد جماليان' •، محسن اسلامدوست"، على رضايى'، شعبانعلى عليزاده" \\ 1. كروه يزشكى قانونى، دانشكده يزشكى، دانشكاه علوميزشكى اراك، اراك، ايران.

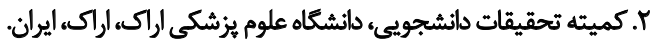

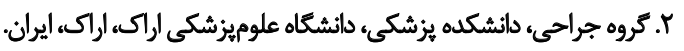

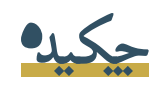

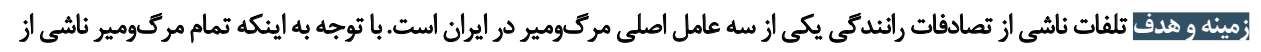

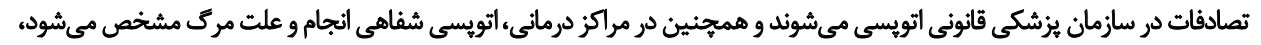

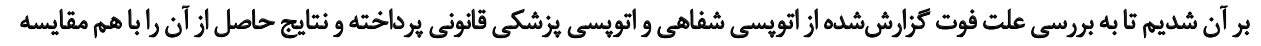
كنيمب

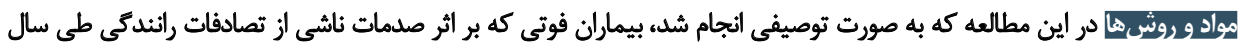

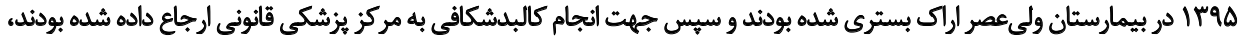

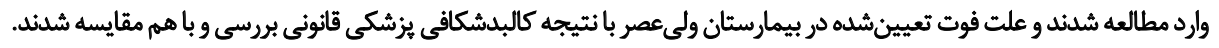

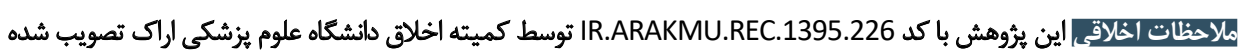

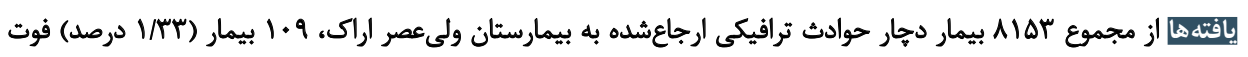

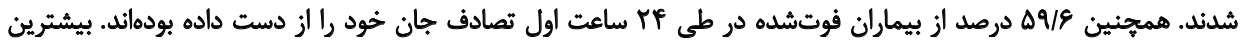

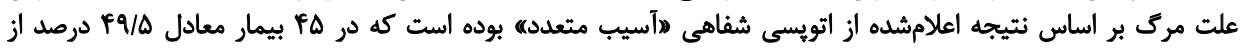
بيماران وجود داشته است.

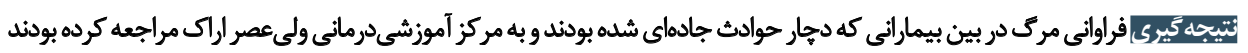

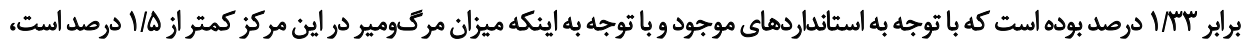

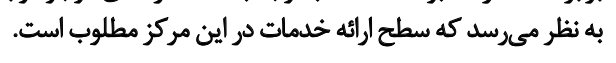

اطلاعات مقاله:

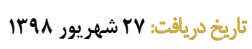
تاريخ بذيرش: 9. خرداد

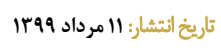

: كليulg

صدمات ترافيكي جاده

اي، مرك، تصادف ترافيكي

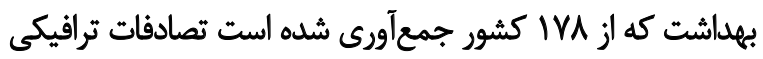

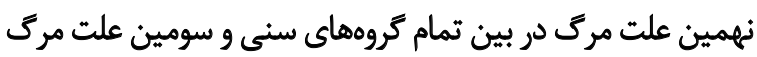

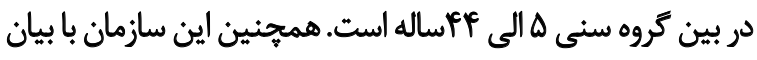

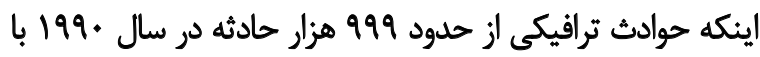

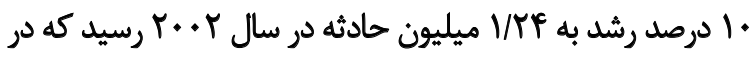

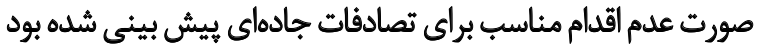

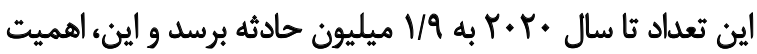

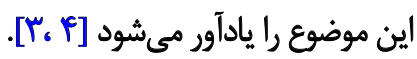
مرك هاى ناشى از حوادث ترافيكى به آن دسته از مرك آدهايى كفته

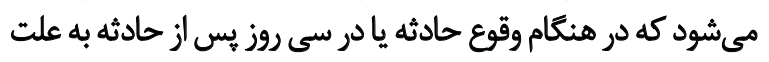

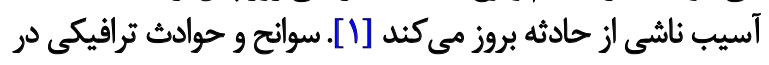

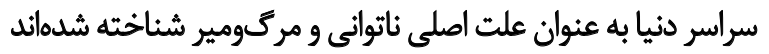

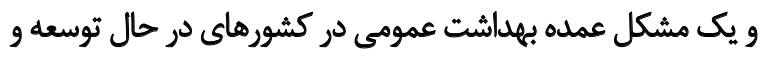

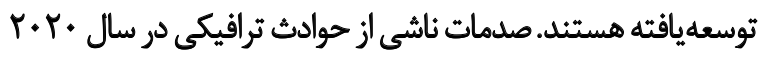

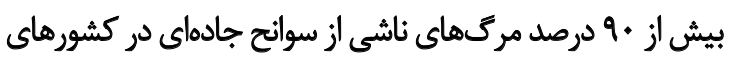

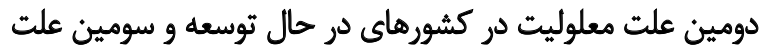

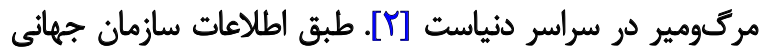
- 


\section{مواد وروشها}

در اين مطالعه كه به صورت توصيفىمقطعى انجام شد، بيماران

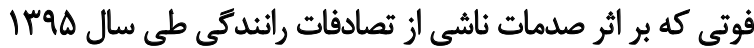

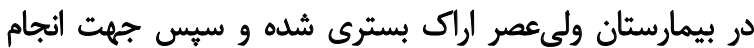

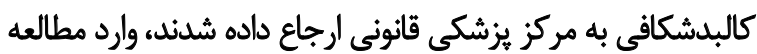

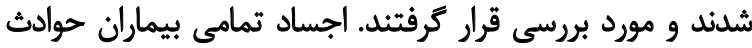
ترافيكى تحت اتويسى يزشكى قانونى قرار مى ثي تريرند.

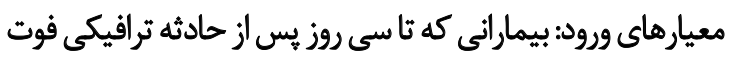

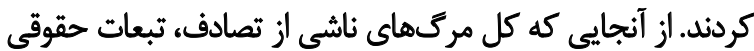

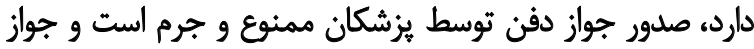

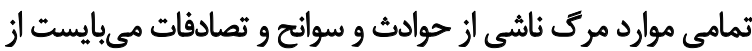

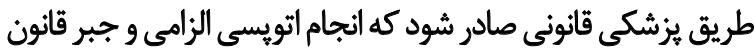

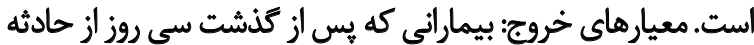

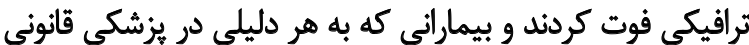
اراك براى آنها اتويسى انجام نشده است وني

صدمات ناشى از تصادفات رانندگى به آن دسته از حوادثى اطلاق

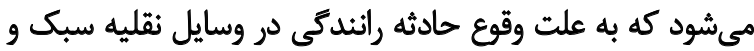

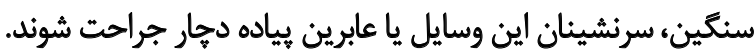

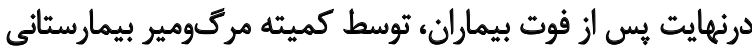

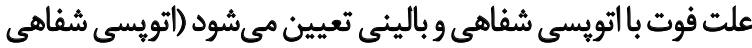

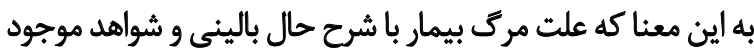

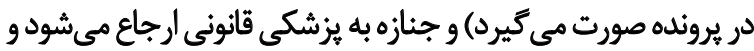

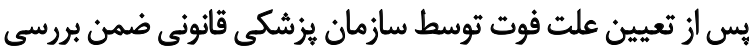

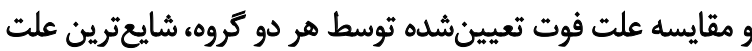

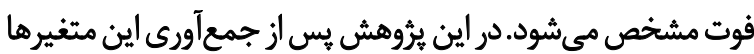

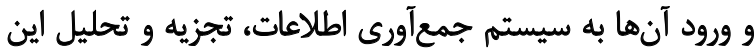
اطلاعات با استفاده از نرمافزار آمارى SPSS انجام شد.

يافتهها

مطالعه اخير با هدف بررسى علل فوت در بيماران حوادث

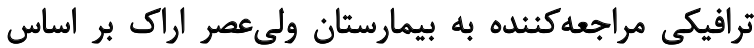

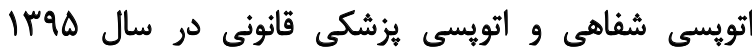

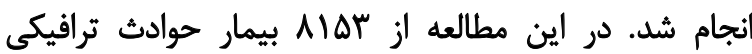

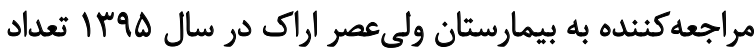

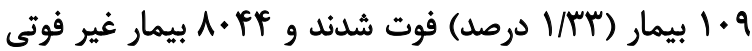

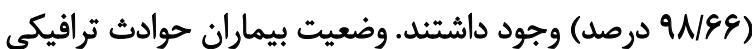

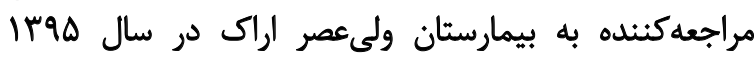

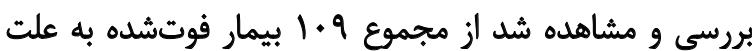

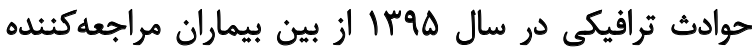

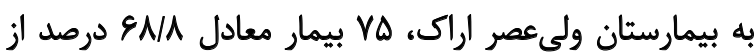

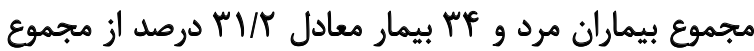

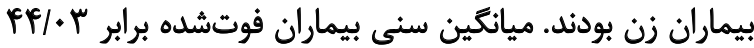

در حال توسعه با درآمد كم و متوسط نح مي دهد. با وجود اينكه

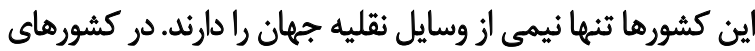

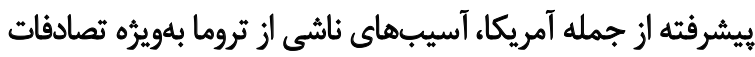

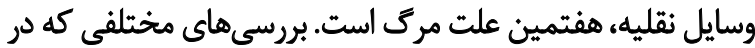

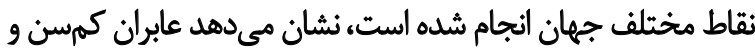

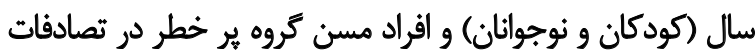

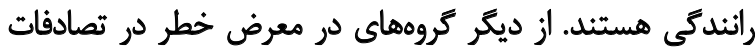

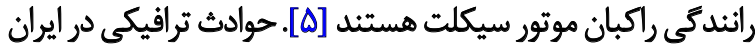

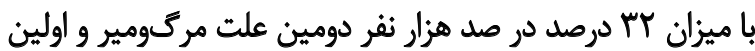

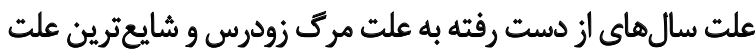

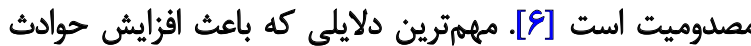

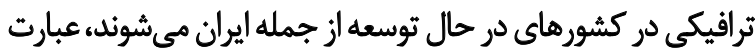

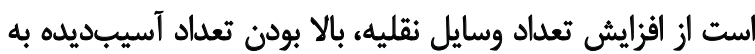

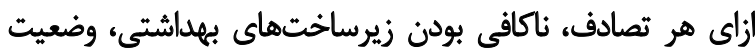

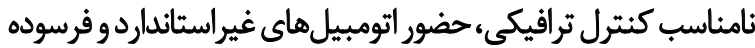

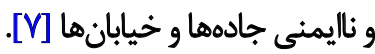

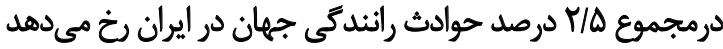

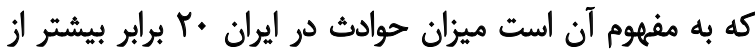

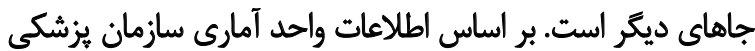

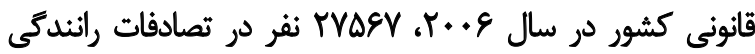

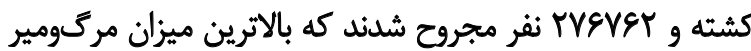

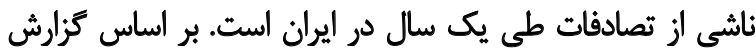

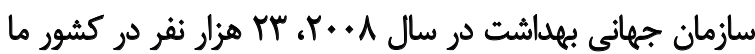

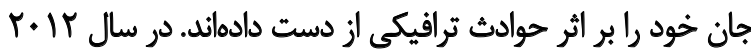

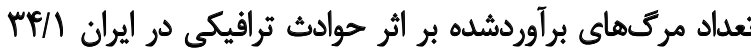

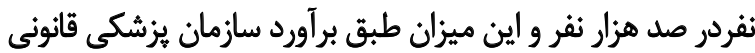

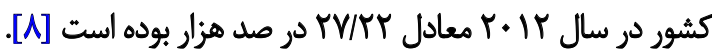

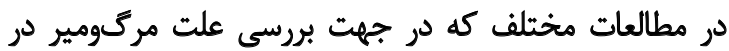

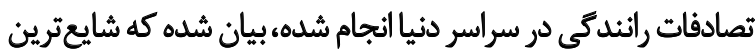

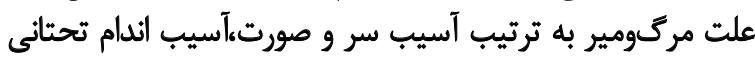

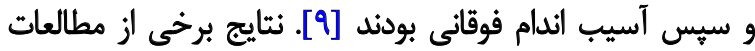

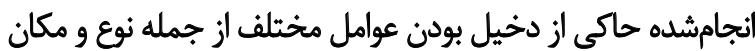

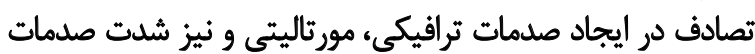

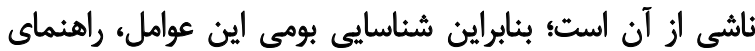

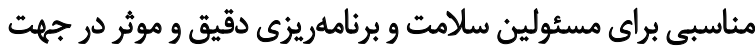

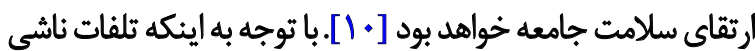

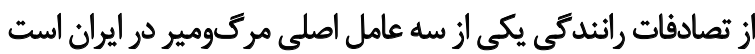

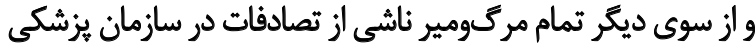

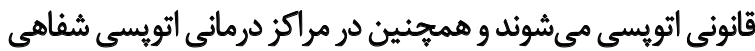

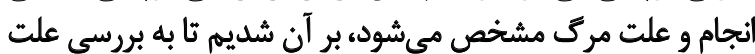

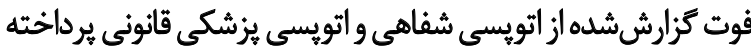
و نتايج حاصل از آن را با هم مقايسه كنيم. 
جدولا. بررسى علت مرك براساس اتويسى شفاهى بيماران

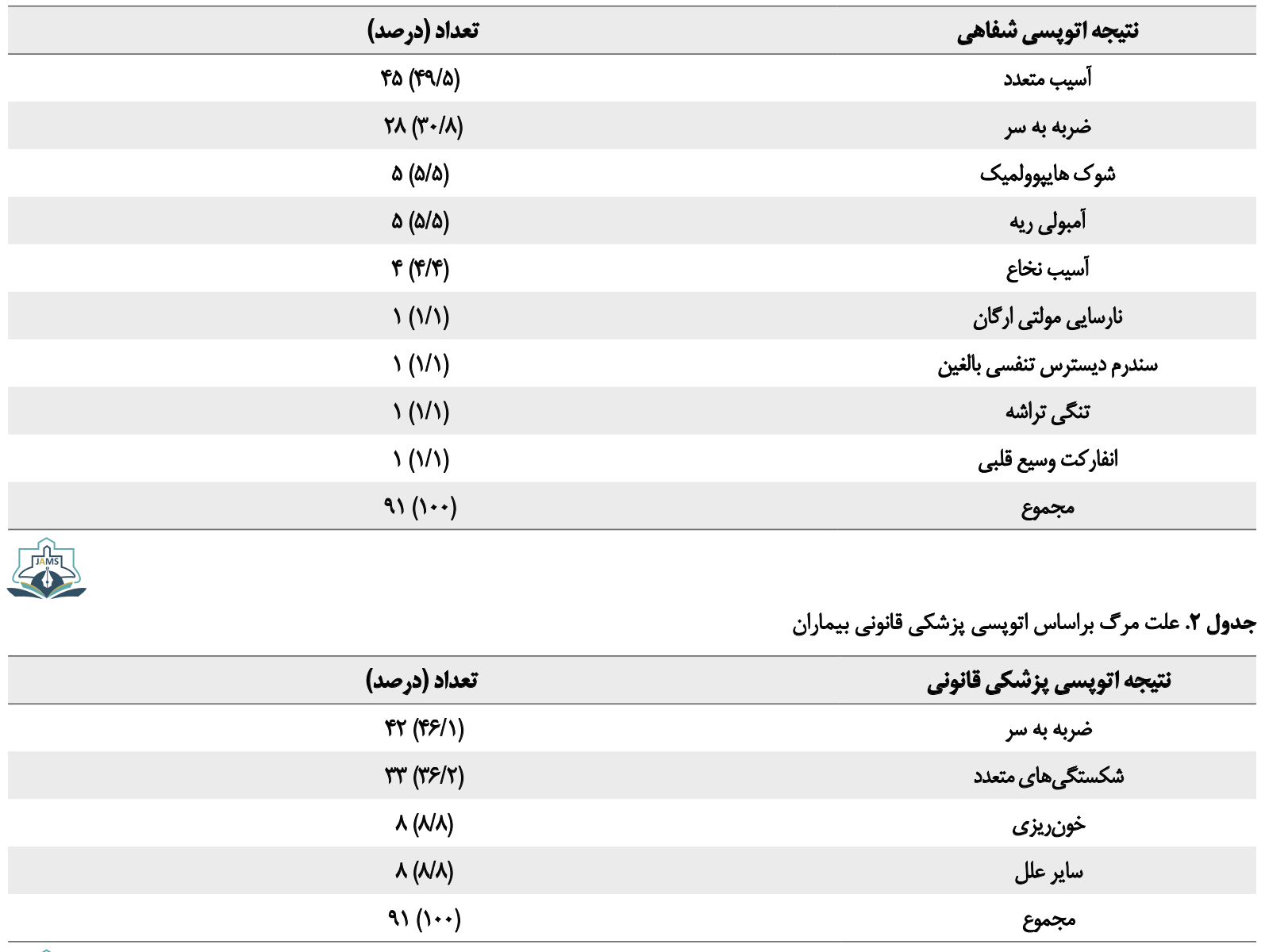

بيماران وجود داشته است. بعد از اين آسيب، دومين علت شايع مرت

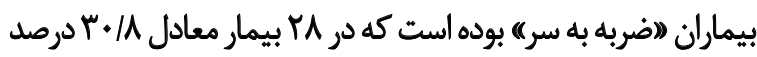

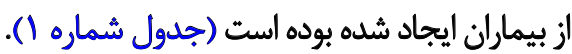

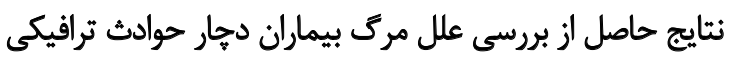

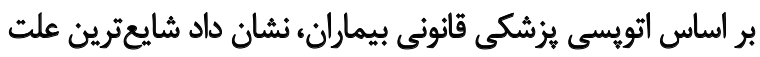

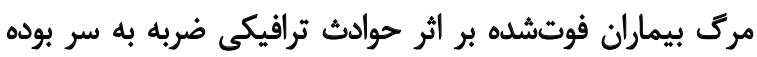

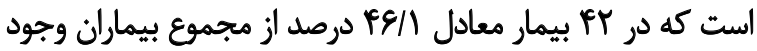

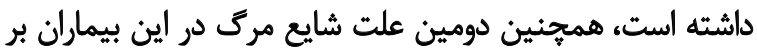

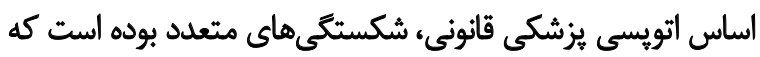

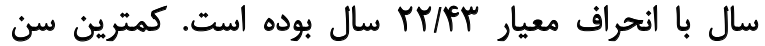

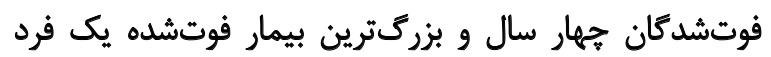

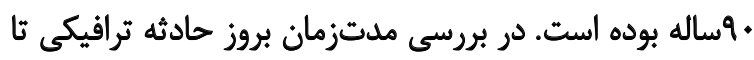

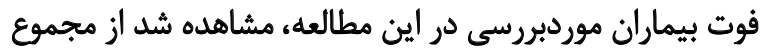

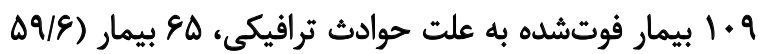

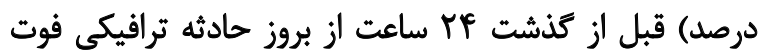

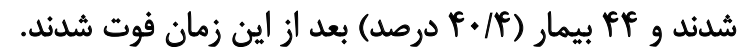
بيشترين علت مرك بر اساس نتيجه اعلامشده از اتويسى شفاهى

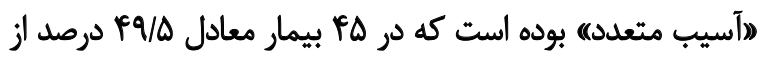
جدول ب. مقايسه علل مركى بيماران براساس اتويسى يزّكى قانونى و اتويسى شفاهى

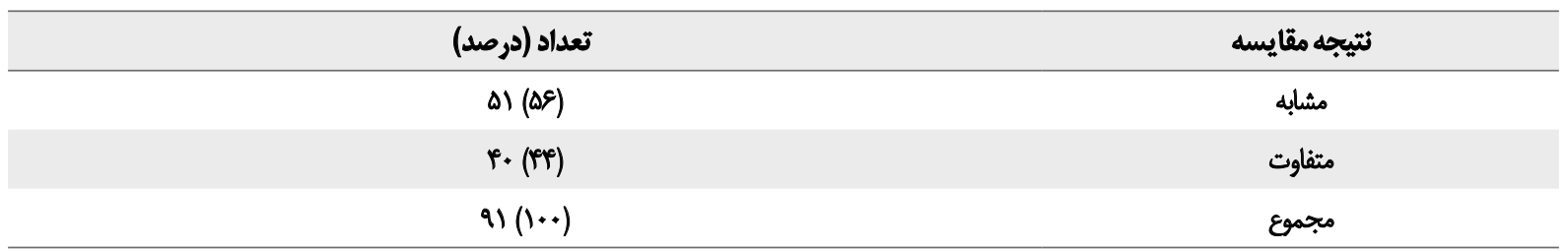


سالاثه بيش از / إميليون نفر در سراسر جهان بر اثر حوادث

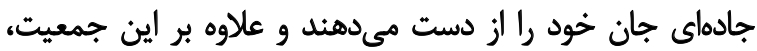

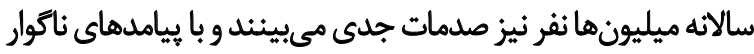

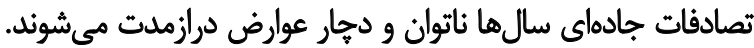

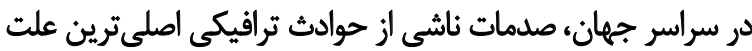

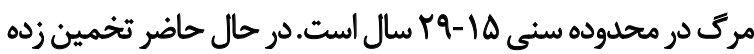

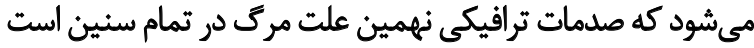

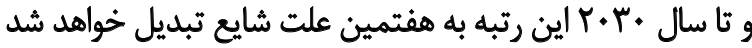

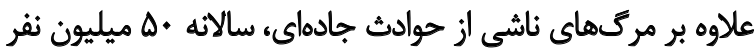

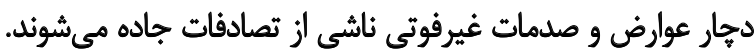

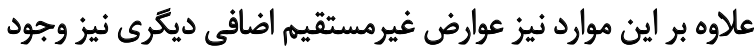

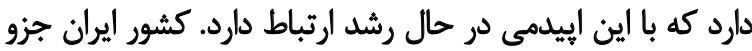

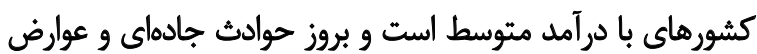

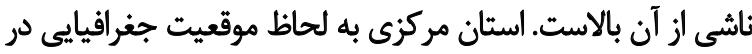

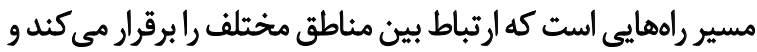

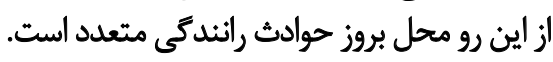

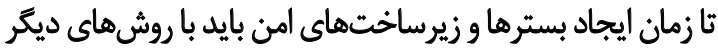

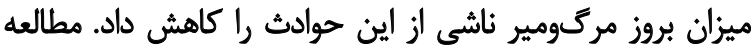

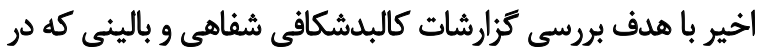

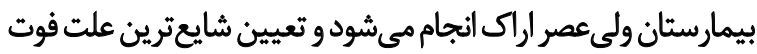

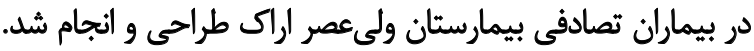

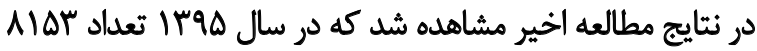

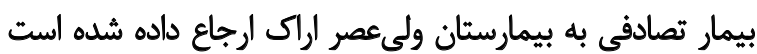

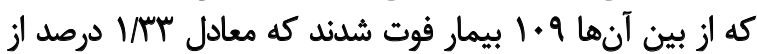

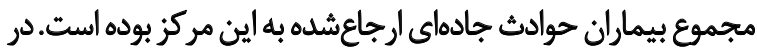

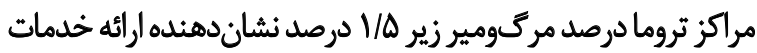

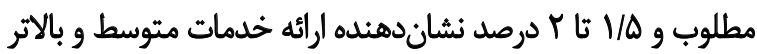

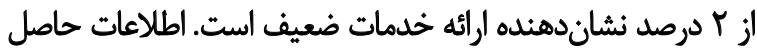

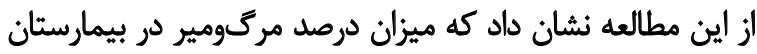

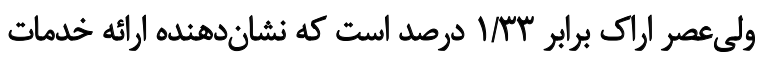
مطلوب در اين مركز است.

در نتايج مطالعه اخير مشاهده شد از بين بيمارانى كه بر اثر إنر

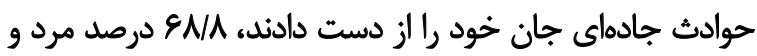

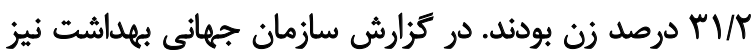

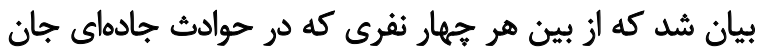

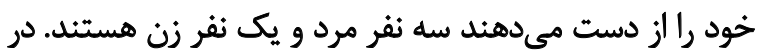

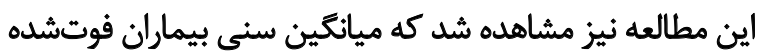

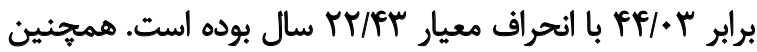

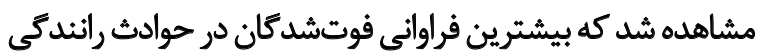

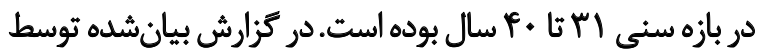

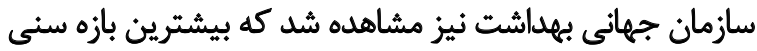

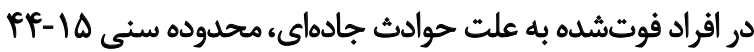

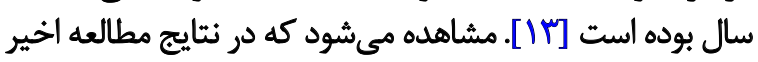
نيز نتايج مشابهى وجود داشته است.در نتايج اين مطالعه مشاهده

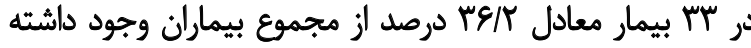

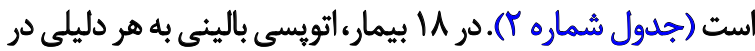

$$
\text { يزشكى قانونى انجام نشده بوده است. }
$$

مقايسه علل فوت بيماران حوادث ترافيكى بر اساس اتويسى

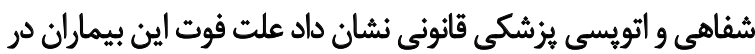

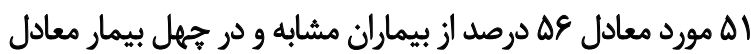

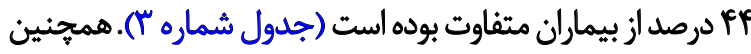

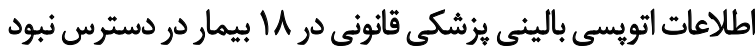

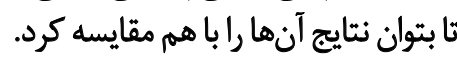

بحث

از الز الم بيمار حوادث ترافيكى مراجعلكنينده به بيمارستان

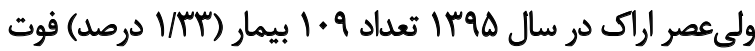

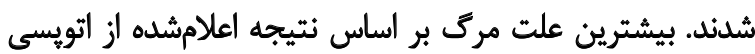

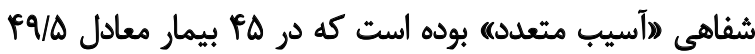

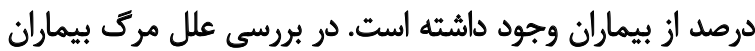

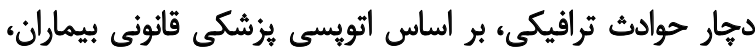

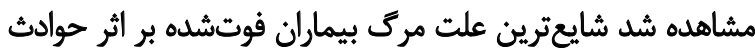

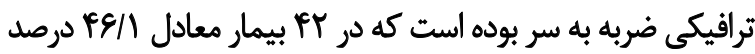
از مجموع بيماران وجود داشته است.

بررسى علل فوت بيماران حوادث ترافيكى بر اساس اتوبسى

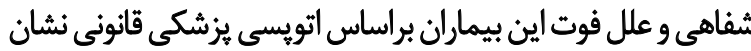

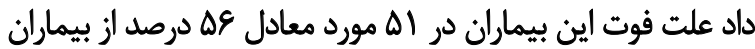

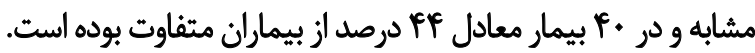

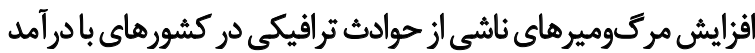

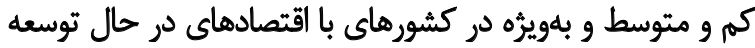

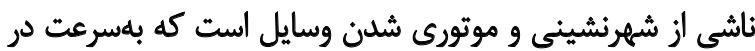

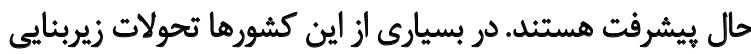

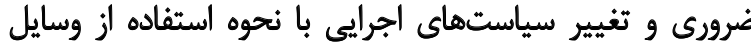

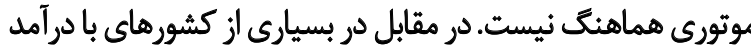

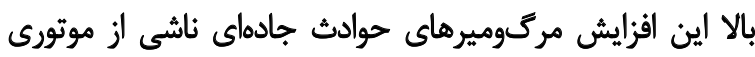

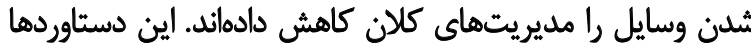

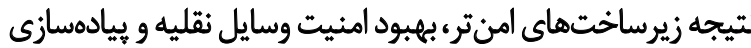

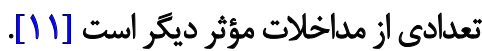

همزمان با افزايش تعداد وسايل نقليه در كشورها، دولتها با بان

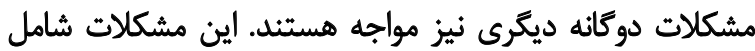

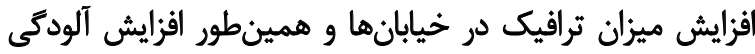

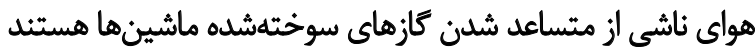

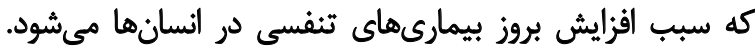

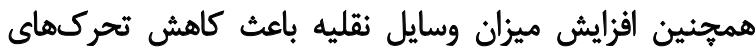

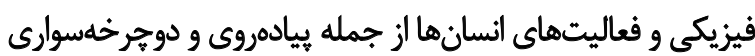

مي شود كه با كاهش سلامتى ارتباط دارد [ [IT]. 


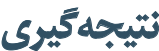

بر اساس نتايجى كه از مطالعه اخير حاصل شد، نتيجهكيرى

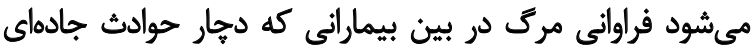

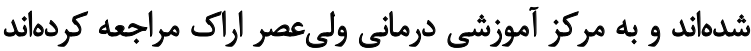

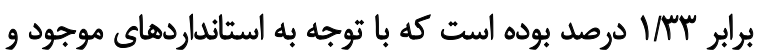

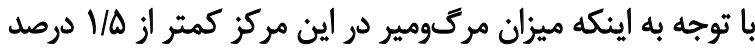

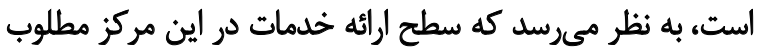

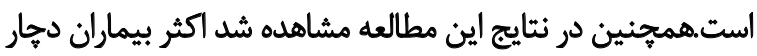

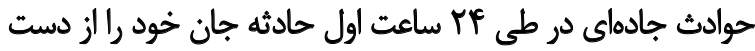

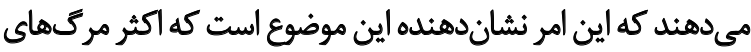

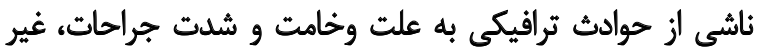

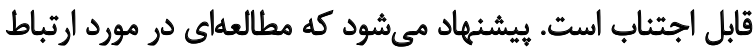

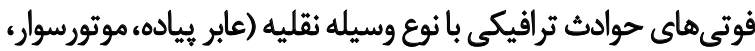

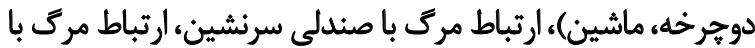

وضعيت تحصيلى و شغل و وضعيت فرهنكيى متوفيان اندين انجام كيرد.

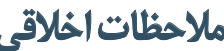

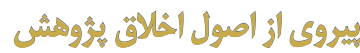

اين يثوهش با كد كميته اخلاق دانشكاه علوم يزشكى اراك تصويب شده است.

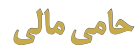

اين يروهش توسط معاونت تحقيقات وفناورى دانشًا علوميزشكى اراك انجام حمايت مالى شده است.

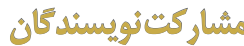

مفهومسازى و مقايسه نتايج: محمد جماليان؛ طراحى يثروهش

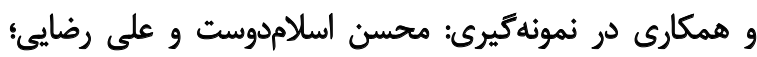
نمونه كيرى: شعبانعلى عليزاده؛ بازبينى متن: تمام نويسندكان.

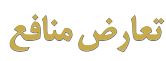

نويسندكان مقاله هيجگونه تعارضى در منافع اعلام نكردند.

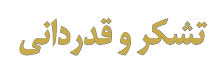

از معاونت تحقيقات و فناورى دانشكاه علوميزشكى اراك و وائيكاه توسعه تحقيقات بالينى بيمارستان ولى الىصر (عج) تشكر و قدردانى

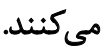

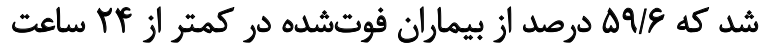

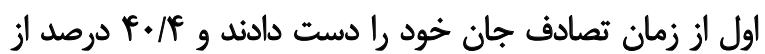
بيماران فوتشده بعد از اين زمان از دنيا رفتند. در مطالعداى كه توسط آريوند كومار و همكارانش انجام شد نيز

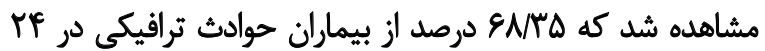

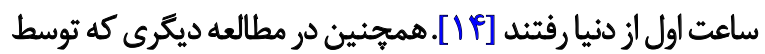

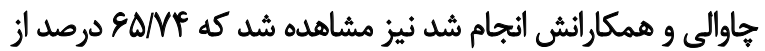

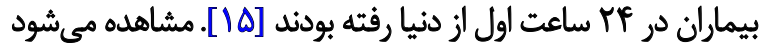

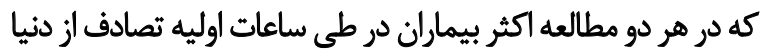

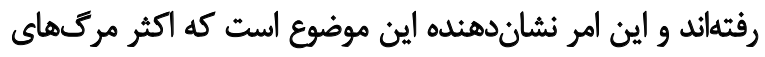

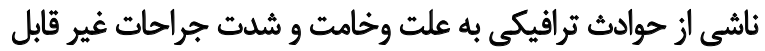

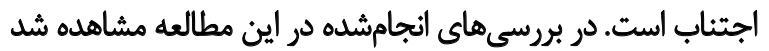

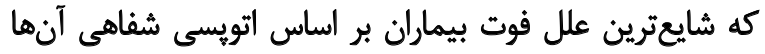

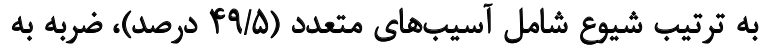

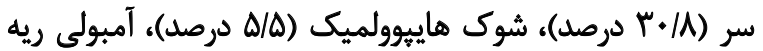

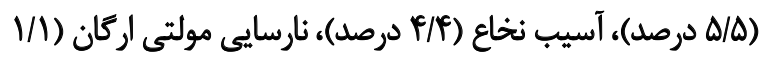

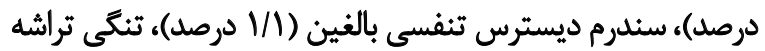
(1/ ادرصد) و انفاركت وسيع قلبى (1/ الدرصد) بود.

در بررسى علت مرك بر اساس اتويسى بزشكى قانونى مانى مشاهده

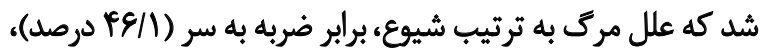

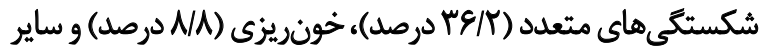

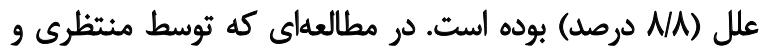

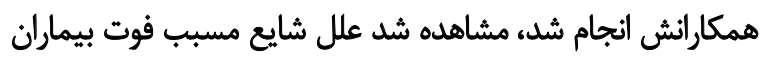

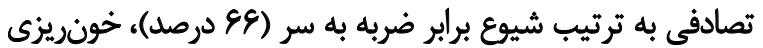

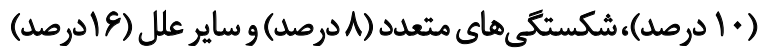

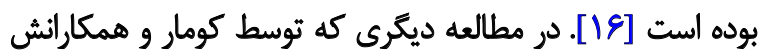

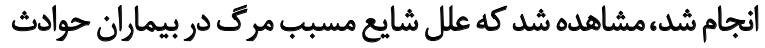

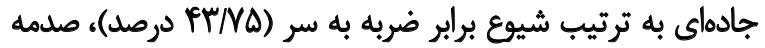

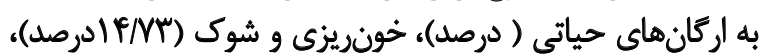

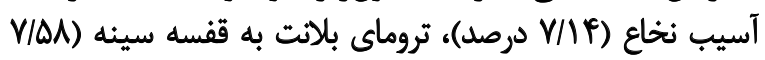

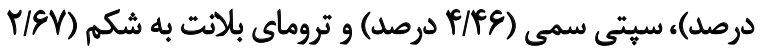

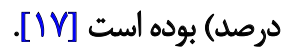

در مطالعه ديكرى نيز كه توسط فروغى و همكارانش انجام شد،

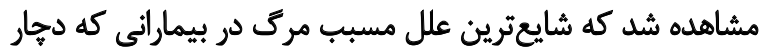

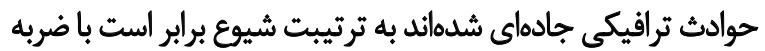

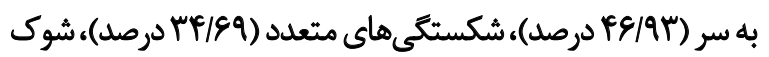

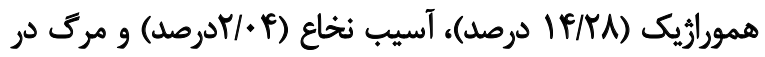

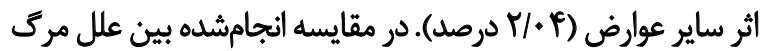

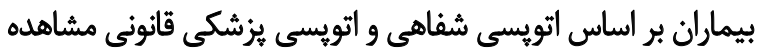

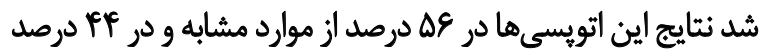

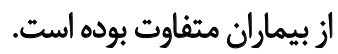




\section{References}

[1] World Health Organization. Global status report on road safety 2009 [Internet]. 2009 [Updated 2009]. Available from: https://www.who.int/ violence_injury_prevention/road_safety_status/2009/en/

[2] Garg N, Hyder AA, Exploring the relationship between development and road traffic injuries: A case study from India. Eur J Public Health. 2006; 16(5):487-91. [DOI:10.1093/eurpub/ckI031] [PMID]

[3] Bakhtiyari M, Soori H. [Epidemiology of traffic crashes outcomes and related factors in Iran 2010 (Persian)]. J Saf Promot Inj Prev. 2013; 1(3):1509. https://www.sid.ir/fa/journal/ViewPaper.aspx?ID=224333

[4] Ziyab A, Akhtar S. Incidence and trend of road traffic injuries and related deaths in Kuwait: 2000-2009. Injury. 2012; 43(12):2018-22. [DOI:10.1016/j.injury.2011.09.023] [PMID]

[5] Ahahla A, Charehsaz S. Injuries iesulting from motorcycle-induced trauma during two years in Shahid Motahari Cinical Center of Urmia. Sci J Forensic Med. 2006; 12(2):79-83.

[6] Khorami Z, Hashemi Nazari S, Ghadirzadeh MR. [An epidemiology study of deaths from road traffic accidents (Persian)]. J Saf Promot Inj Prev. 2017; 4(4):217-24. https://www.sid.ir/en/journal/ViewPaper. aspx?id=522442

[7] Hashemi Nazari SS, Kazemian M, Hosseini F. [Trend of five years traffic accident mortality in khuzestan province (2006-2010) (Persian)]. Sci J Forensic Med. 2011; 17(2):123-9. https://www.sid.ir/en/journal/ViewPaper.aspx?id=285222

[8] Mobaleghi J, Molanaei NA. Evaluation of death and injuries caused by traffic accidents in patients admitted to Besat Hospital of Sanandaj in 1380. Sci J Kordestan Univ Med Sci. 2003; 7(2):28-30. https://www.sid. ir/fa/journal/ViewPaper.aspx?id=12721

[9] Nazari R, Bijani A, Haji Hosseini F, Beheshti Z, Sharifnia SH, Hojati H. [Mortality and Injury severity in the accident victims referred to the Hefdah Shahrivar hospital of Amol 2007 (Persian)]. J Babol Univ Med Sci. 2011; 13(158):76-81. http://jbums.org/article-1-3722-en.html

[10] Davoodi F, Ghadirzadeh M. [Epidemiology Study of Road Traffic Accidents Resultingin Death: In Lorestan Province in 2012 (Persian)]. J Saf Promot Inj Prev. 2016; 3(4):257-62. https://www.sid.ir/en/Journal/ ViewPaper.aspx?ID=509426

[11] World Health Organization. World report on road traffic injury prevention. Geneva: World Health Organization; 2004. https://b2n.ir/406912

[12] World Health Organization. Global health risks: Mortality and burden of disease attributable to selected major risks. Geneva: World Health Organization; 2004. https://apps.who.int/iris/handle/10665/44203

[13] World Health Organization. Global status report on road safety, Time for Action. Geneva: World Health Organization; 2004. http://whqlibdoc. who.int/publications/2009/9789241563840_eng.pdf

[14] Kumar A, Lalwani S, Agrawal D, Rautji R, Dogra TD. Fatal road traffic accidents and their relationship with head injuries: An epidemiological survey of five years. Indian J Neurotrauma. 2008; 5(2):63-7. [DOI:10.1016/ S0973-0508(08)80002-0]

[15] Chavali KH, Sharma BR, Harish D, Sharma A. Head injury: The principal killer in road traffic accidents. J Indian Acad Forensic Med. 2006; 28:1214. http://iafmonline.in/data/publications/2006/JIAFM-28(4).pdf

[16] Montazeri A. Road-traffic-related mortality in Iran: A descriptive study. Public Health. 2004; 118(2):110-3. [DOI:10.1016/S00333506(03)00173-2]
[17] Kumar N, Ghormade PS, Tingne CV, Keoliya AN. Trends of fatal road traffic accidents in central india. J Forensic Med, Sci Law. 2013; 22(2):811. https://www.researchgate.net/publication/303882875_Trends_of_ Fatal_Road_Traffic_Accidents_In_Central_India 
This Page Intentionally Left Blank 